\title{
Age and Mass of the Local Group from Satellite Galaxy Dynamics
}

\author{
Alan B. Whiting \\ Physics Department, U. S. Naval Academy, Annapolis, MD 21402, USA
}

\begin{abstract}
.
The present kinematics of an unrelaxed system such as the Local Group can be used to investigate its dynamics on a cosmological time scale. In particular, the classical timing argument of Kahn \& Woltjer may be extended to distant satellite galaxies of the Local Group whose distances and velocities are known with some accuracy. Including a correction for the fact that the Local Group was not always dynamically isolated, I derive a mass for the Group of about $1.6 \times 10^{12} M_{\odot}$ and an age of 1.0 to $1.2 \times 10^{10}$ years.
\end{abstract}

\section{Introduction}

The universe is a very large and complicated place. For a cosmologist this is unfortunate, for it makes calculations difficult; hence the universe is usually assumed, at some level of approximation, to be small and simple. One example of this is the timing argument of Kahn \& Woltjer (1959), in which the motions of the Milky Way and M31 were considered as a one-dimensional, two-body problem, the rest of the universe being ignored. From this they derived a number made up of the age of the pair (assumed to be the same as the age of the universe) and their total mass; given one, the other quantity could be found.

As pointed out by Lynden-Bell (1981), distant dwarf galaxies in the Local Group can be used to extend this method. Not only are more data points then available to refine and check its accuracy, galaxies at different phases of their trajectories can separate age from mass and so allow each to be determined independently.

\section{Calculations and Complications}

Figure 1 displays the geometry of the situation and some of the assumptions involved. The Milky Way and Andromeda, with most of the mass of the Local Group, are assumed to act as one body located at the centre of mass. Each satellite galaxy is assumed to be on a one-dimensional orbit on a line directly away from that centre. From our position, we see the projection of its actual velocity along our line of sight. All satellites are assumed to be of negligible 


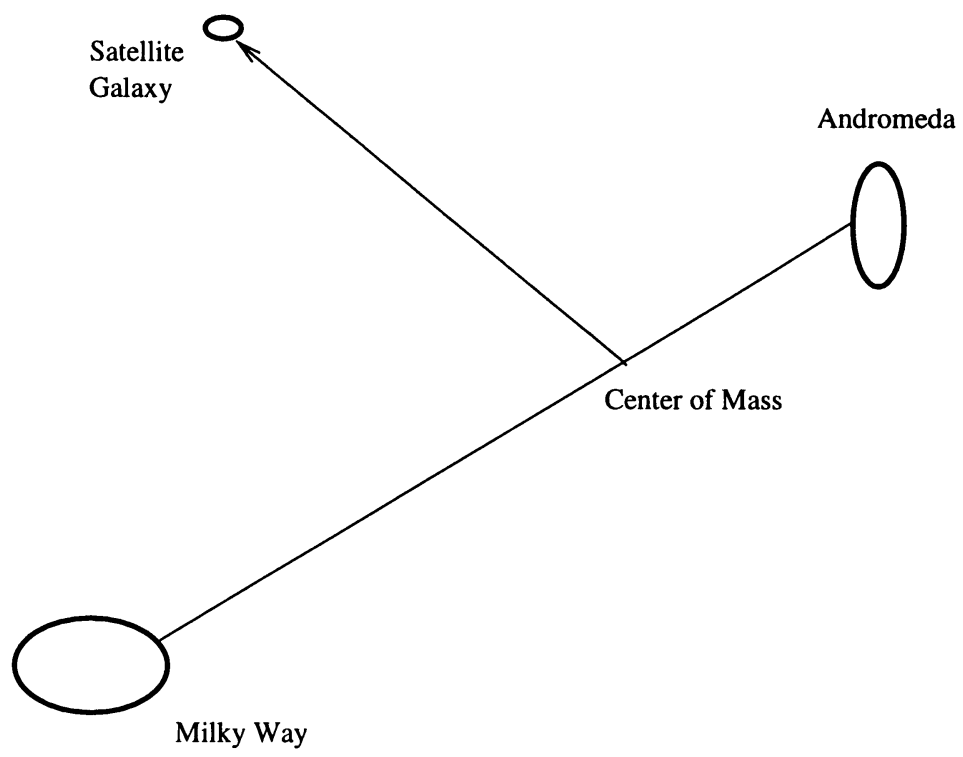

Figure 1. The geometry of the satellite galaxy calculation. Each distant dwarf galaxy of the Local Group is assumed to be on a radial trajectory away from (or toward) the centre of mass of the Group, which is assumed to lie on a line from the Milky Way to M31 at twothirds the distance. The observed radial velocity is then the projection of this radial motion along our line of sight.

mass, and thus their distance $a$ from the centre of mass obeys the equation

$$
\ddot{a}=-\frac{2 G M}{a^{2}}
$$

where $2 M$ is the total mass of the two major galaxies (and thus, by assumption, of the Local Group).

There are four immediate complications to this simple picture. First, the distance to M31 is uncertain by a significant amount. When Lynden-Bell (1981) performed his calculation, $680 \mathrm{kpc}$ seemed a good value; I have chosen the more recent figure of $725 \mathrm{kpc}$. Later determinations have given $800 \mathrm{kpc}$ and, at this symposium, Michael Feast has quoted a $900 \mathrm{kpc}$ result. For the most part, however, this uncertainty in distance makes little difference to the geometry of Fig. 1, and is anyway at least partially masked by the uncertain ratio of the mass of M31 to the Milky Way (taken here as 2:1). For the present calculation I ignore the problem. 
Second, the Milky Way and M31 are two masses, not one, which fact could significantly modify satellite motions. I minimise this effect by choosing satellites distant from both major galaxies, later investigating a correction a posteriori.

Third, to reduce the observed heliocentric radial velocity to the velocity with respect to the Milky Way (and eventually to the centre of mass of the Local Group), the circular velocity of the sun around the Milky Way must be known. Estimates have ranged from 180 to $300 \mathrm{~km} \mathrm{~s}^{-1}$, with some tendency to settle on $220 \mathrm{~km} \mathrm{~s}^{-1}$ as a compromise (by no means universally accepted). I treat this as another parameter to be adjusted for a good fit, calculating results for a wide range of velocities.

Last, the Local Group has not always been dynamically isolated. At some point in the early history of the universe other galaxies, now distant, were close enough to have significant effects. Earlier still, all matter would have been more or less smoothly distributed, thus obeying the equation of motion for a continuous fluid under gravity:

$$
\ddot{a}=-\frac{4 \pi G}{3} \rho a
$$

with $a$ the distance between any two points, and $\rho$ the mass density. For $\rho \propto$ $a^{-3}$ this equation and equation (1) are of the same form, which is comforting; unfortunately, the coefficients are not the same. How much $4 \pi G \rho / 3$ differs from $2 G M$ is a difficult question to answer, depending on how nearby protogalaxies fit together immediately after they started to collapse. Trying to maintain what simplicity is left in the calculation, I assumed a simple cubic lattice of masses $M$, which leads to a difference of $2 \pi / 3$, slightly more than double. That is, two bodies which actually move as parts of this lattice, but whose combined mass is calculated as if they were isolated, will yield a total mass too high by a factor of about 2.09 .

Of course, the Local Group is a collapsing, over-dense region, not part of an infinite lattice of uniform overall density. Equation (2) must apply at the beginning, and something like equation (1) now. To interpolate between them, I use the equation

$$
\ddot{a}=-\frac{2 G M}{a^{2}}\left(1+\left(\frac{2 \pi}{3}-1\right) \frac{a^{3}}{b^{3}}\right)
$$

with $a$ the distance between two bodies and $b$ the corresponding distance between two bodies expanding along with a critical $\left(b \propto t^{2 / 3}\right)$ universe. This equation was solved numerically for a range of masses and phases of the orbit, and the resulting curves plotted for comparison with data.

\section{Plots and Data}

Satellite galaxies with published distances and radial velocities were chosen for inclusion in the plot, based on distance from both the Milky Way and M31. For each plot an age of the Local Group and a circular velocity of the Sun around the Milky Way were chosen, which (with the geometry as shown in Fig. 1) yielded the values $a$ (in Mpc) and $\dot{a}$ (in Mpc per age of the Group). These were plotted against the derived curves of constant $G M$. From this plot the average $G M$ and 
the dispersion (in $\mathrm{kpc}$ ) about the average were calculated, ignoring those galaxies which plotted above or to the left of the curves (these are not bound, according to the assumptions of the model, so their dynamics would not necessarily be determined by the Local Group alone). The plot was done for values of the circular velocity of the sun from $200 \mathrm{~km} \mathrm{~s}^{-1}$ to $260 \mathrm{~km} \mathrm{~s}^{-1}$ (at intervals of 20 $\mathrm{km} \mathrm{s}^{-1}$ ) and for ages of 8, 10, 12 and 14 Gyr.

The plot with the smallest dispersion used $220 \mathrm{~km} \mathrm{~s}^{-1}$ and $10 \mathrm{Gyr}$. (Slightly larger dispersions were given by 200 and $240 \mathrm{kms}^{-1}$, and $12 \mathrm{Gyr}$; for an age of less than 10 Gyr the dispersion rises steeply.) The resulting mass of the Local Group was $1.4 \times 10^{12} M_{\odot}$. Unfortunately, this value is about half that given by the Milky Way-M31 pair for the same circular velocity and age. Since we might expect the two major galaxies to approximate a two-body system more accurately than smaller (more easily deviated) galaxies, it is worthwhile to see whether agreement between the satellites and the major galaxies can be obtained. For a circular velocity of $240 \mathrm{~km} \mathrm{~s}^{-1}$ the satellite galaxy dispersion about the mean is not significantly greater, and M31 agrees with them in a mass of $1.6 \times 10^{12} M_{\odot}$. The resulting plot is shown in Fig. 2 .

For comparison, a set of curves using only the two-body formula (equation 1) was constructed and the same procedure followed. The dispersion about the mean curve, for a solution using $10 \mathrm{Gyr}$ and $240 \mathrm{~km} \mathrm{~s}^{-1}$, was not significantly different from that found using the interpolated formula (equation 3); and the mass found was more in line with other recent determinations at $3.8 \times 10^{12} M_{\odot}$. However, for two reasons the two-body formula is probably less accurate. First, the minimum dispersion is a very shallow minimum, the dispersion not varying significantly over the range studied. This suggests that the model does not fit the data well. Second, the actual minimum of the dispersion occurs for an age of $8 \mathrm{Gyr}$. While it is conceivable that stellar ages might allow an age of 12 or even $10 \mathrm{Gyr}, 8$ is beyond the limits of uncertainty.

\section{Corrections and Uncertainties}

The fact that a number of the more distant dwarf galaxies in the Local Group do fall close to the $a-\dot{a}$ curve for the simple spherical model indicates that the model is useful as a first approximation for the dynamics of the Group.

Returning to the inaccuracy in treating two large galaxies as one mass, correction terms were attempted in the form of a dipole and quadrapole (with the axis along the Milky Way-M31 line). Neither resulted in an improvement. This does not mean that the spherical expansion model is necessarily an accurate picture of Local Group dynamics, only that the next order of improvements is not the obvious one.

The standard deviation is about $180 \mathrm{kpc}$ around the mean. This translates into a factor of about 1.7 above or below the average value of mass, or a factor of three overall. This is not as tight a constraint as one would like. Better distances might help (their uncertainties are about the same size as the standard deviation); but it is unlikely that a major improvement (say a factor of four to ten) will occur very soon. More importantly, though the data points do follow the theoretical curve in general, the dispersion is real, and indicates that the model does not quite describe the dynamical behaviour of the Group 


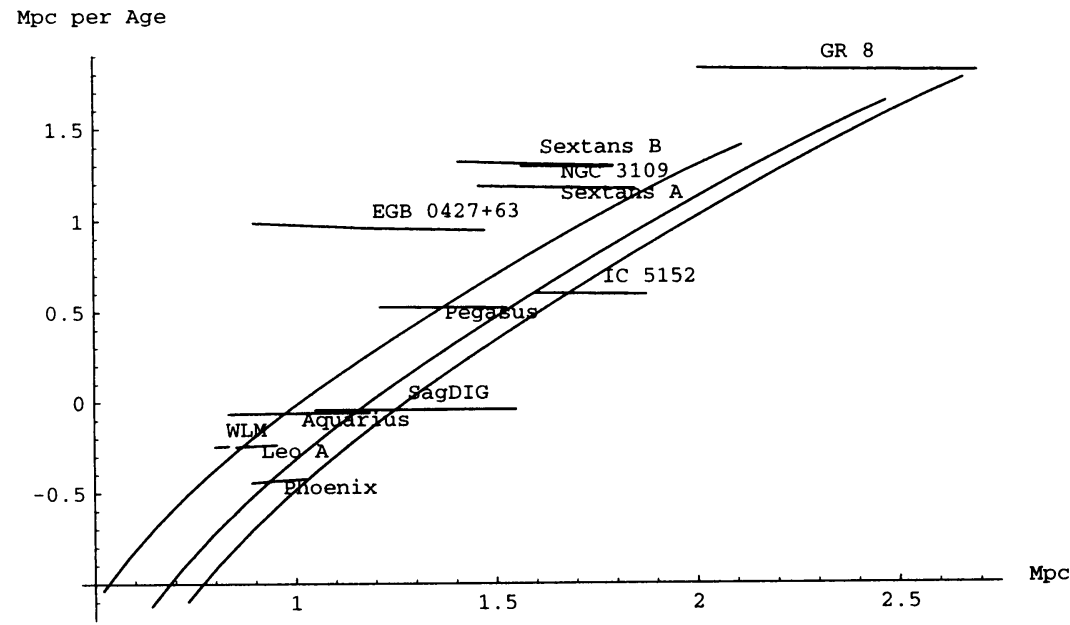

Figure 2. The plot of least dispersion, using an age of $10 \mathrm{Gyr}$ and a circular velocity of the sun around the Milky Way of $240 \mathrm{~km} \mathrm{~s}^{-1}$. The three curves shown give a mass for the Local Group, from left to right, of $1.2 \times 10^{12}, 1.8 \times 10^{12}$, and $2.3 \times 10^{12} M_{\odot}$. Distance and radial velocity data for the dwarf galaxies were taken from: GR8, Hoessel \& Danielson (1983), Hodge (1974); EGB 0427+63, Hoessel et al. (1988), Van den Bergh (1994); Leo A, Tolstoy et al. (1998); Aquarius, Lee, this conference; IC 5152, Caldwell et al. (1988); Pegasus, Sandage (1986), Hoessel et al. (1990); WLM, Sandage \& Carlson (1985b), Minniti \& Zijlstra (1996); Sextans A and B, Sandage \& Carlson (1985a), Tosi et al. (1991); NGC 3109, Lee (1993); Phoenix, Schmidt \& Boller (1992), Caldwell et al. (1988); SagDIG, Van den Bergh (1994). Uncertainties were not given in the sources for EGB 0427+63, IC 5152 and NGC 3109, so estimated errors are plotted which are similar to others listed. 
within existing uncertainties. Probably the next step is to take into account the specific influences of the nearby groups of galaxies, a much more complicated undertaking.

Acknowledgements. The author wishes to thank Donald Lynden-Bell for encouragement and suggestions during the calculations, and Lisa Becktold for much help with obscure software.

\section{References}

Caldwell, N., Schommer, R.A., Graham, J.A. 1988, BAAS, 20, 1084

Hodge, P. 1974, PASP, 86, 645

Hoessel, J.G., Danielson, G.E. 1983, ApJ, 271, 65

Hoessel, J.G., Saha, A., Danielson, G.E. 1988, PASP, 100, 680

Hoessel, J.G., Abbott, M.J., Saha, A., Mossman, A.E., Danielson, G.E. 1990, AJ, 100, 1151

Kahn, F.D., Woltjer, L. 1959, ApJ, 130, 705

Lee, M.G. 1993, ApJ, 408, 409

Lynden-Bell, D. 1981, Observatory, 101, 111

Minniti, D., Zijlstra, A.A. 1996, ApJ, 467, L13

Sandage, A. 1986, AJ, 91, 496

Sandage, A., Carlson, G. 1985a, AJ, 90, 1019

Sandage, A., Carlson, G. 1985b, AJ, 90, 1464

Schmidt, K.-H., Boller, T. 1992, Astronomische Nachrichten, 313, 189

Tolstoy, E., Gallagher, J.S., Cole, A.A., Hoessel, J.G., Saha, A., Dohm-Palmer,

R.C., Skillman, E.D., Mateo, M., Hurley-Keller, D. 1998, AJ, 116, 1244

Tosi, M., Greggio, L., Marconi, G., Focardi, P. 1991, AJ, 102, 951

Van den Bergh, S. 1994, AJ, 107, 132

\section{Discussion}

Freeman: Can you put your finger on the main source of why you get a lower mass than Schmoldt \& Saha (12th Potsdam Cosmology Workshop, 1997): is it that you use mainly satellites that are more distant?

Whiting: I believe the main difference lies in the fact that I force the dynamics to fit onto a continuous-fluid solution at the beginning, which leads to a lower mass estimate (at the extreme, by a factor of two to three).

Lynden-Bell: If there was a bath of undifferentiated dark matter centrally symmetrically bathing the Local Group - would you have measured its mass - i.e., would its mass be already included in the answer or would it have to be added?

Whiting: The current formulation is not quite of the proper form to account for an extended dark halo, although it would in some sense be measured by this 
method.

Zaritsky: Have you had a chance to apply your method to analogs of the Local Group drawn from cosmological N-body simulations to check whether there is any systematic bias in the mass estimate?

Whiting: I've not applied this method to any N-body simulations. It is a very good idea and I'll look into it.

Terndrup: Can your method incorporate proper motions?

Whiting: Unfortunately, the only galaxies with proper motions (or that will have proper motions in the foreseeable future) are much too close to treat by this method.

Hesser: Can you point to those data or changes that you feel would lead to the most significant improvements in your analysis? For instance, we've heard quite a bit just today about the difficulties of assigning membership to the Local Group or Sculptor Group.

Whiting: We already have examples of the sort of observational data that would help, in Lee's determination of a distance to Aquarius (DDO 220) and Tolstoy's distance to Leo A, both presented at this conference. It would also help if more satellite galaxies were found at larger distances, to populate the sparser region of my plot.

However, the most significant limitations of the method lie in the very simple picture it assumes of Local Group motion. To treat the motion with any increased accuracy would require including the nearby groups (Sculptor, M81, Centaurus, etc.) explicitly, which would be much more complicated and difficult (it is, however, the next obvious step). 